\title{
CONCIENCIA BÁSICA
}

\author{
Julio Alvites Ramirez ${ }^{1}$ \\ Universidad Nacional Mayor de San Marcos, Perú \\ (Recibido el 04/09/2007, aceptado el 25/09/2007)
}

\section{RESUMEN}

La conciencia en la Teoría Psicobiológica social dialéctica, de Pedro Ortiz Cabanillas, es la información social codificada en el neocórtex, que se traduce como información psíquica consciente. Su estructuración y organización depende de la cantidad y calidad de información social que refleje el sujeto, lo que permite identificar el grado de desarrollo conciencial. Hay información social que permite y otra que lo impide, una dará solo un cambio o evolución (Información social de evolución-ISE) y otra el desarrollo psíquico y personal del individuo (Información social de desarrollo-ISD).

Por desarrollo se entiende el proceso de socialización, por el cual el humano, recién nacido, va a transformarse en persona y cuya finalidad está en alcanzar la dignidad, la autonomía y la integridad; con la ISD se logrará efectivamente el desarrollo. La ISE es aquella que no contribuye a la formación de esas características personales, por el contrario lo mantiene alejado al individuo de su verdadero derrotero de avance como personalidad, está en mayor cantidad y cualidad en nuestra sociedad y hace que no se pueda mirar lo sustantivo y esencial de la persona: información de los medios de divulgación en su conjunto, muchas veces la propia escuela se convierte en la propagadora de información dogmática, unilateral, inesencial, etc., colocando al individuo dentro de un mundo que no tiene que ver con la realidad propia del individuo. Una conciencia con este grado de desarrollo la denominamos conciencia básica.

Palabras clave: Conciencia, Conciencia básica, Información social, Información social de desarrollo e Información social de evolución.

\begin{abstract}
Doctor's Pedro Ortiz Cabanillas, psychobiologic social dialectic theorie proposes: Conscience is a social encoded information in the neocortex which becomes psychic conscious information. Its structure and organization is a consequence from quality and quantity of social influence given by the "mass media" that human being can reflect, making and showing his consicious developement degree. There's information that inhibits conscious development and gives only a change or evolution (social information of evolution SIE); another allows the psychic and personal development of human being (social information of development- SID).
\end{abstract}

1 Docente Auxiliar de la Facultad de Psicología de la UNMSM. E-mail: jalvitesa@unmsm.edu.pe 
We understand "development" as a socialization process by which, human being becomes Person, and its aim is to reach dignity, authonomy and integrity.

The SID 'S characteristics is to organize the human conscience and its development to reach personality. The SIE inhibits the making of characteristics in human person, keeping it away from its truth path to personality (to be person ).

Here in our society, this kind of information (SIE) "huge" in quantity and quality, inhibits to see the sustantive and essential ways to become human person. It is in the "mass media", that school even spreads, by teaching dogmatic, unessential and only one way information, putting human being away, in another unreal world, far from his own reality.

Conscience with such grade of development we call: Basic conscience.

Keywords: Conscience, Basic conscience, Social information, Social information of evolution, Social information of development

\section{CONCIENCIA}

Para explicar lo que es conciencia básica es necesario entender qué es la conciencia como el sistema psíquico de la personalidad, tomando como punto de partida la teoría psicobiológica dialéctica del Dr. Pedro Ortiz. El ser humano tiene su inicio en el momento de la concepción o sea en la unión del óvulo y el espermatozoide, comenzando así la determinación epigenética, teniendo ésta como eje sustancial a los diferentes tipos de información que determinan la estructura y actividad de los cuatro niveles de la determinación epigenética cuyo primer nivel es el genético, le sigue el tisular, luego el orgánico, hasta el nivel psíquico inconsciente; esta determinación tiene como sustancialidad a los diferentes tipos de información: genética, metabólica, neural y psíquica inconsciente, que organizan la estructura activada propia y singular de cada uno de esos niveles. La información genética organizará y estructurará el nivel genético; la información metabólica hará lo mismo con el nivel tisular; la información neural actuará en el nivel orgánico, $\mathrm{y}$, por último, la información psíquica organizará el nivel psíquico inconsciente. A los nueve meses se produce el nacimiento del niño que trae en su cerebro un neocórtex no estructurado para ser persona, pero con características propias de un humano y que tiene toda la posibilidad de llegar a ser aquella. Es con la información social, que refleja el recién nacido, que se va a dar otro tipo de determinación, llamada cinética, expresada en la interacción del individuo con la sociedad y que se le denomina específicamente sociocinesis. Durante este proceso la información social, al reflejarse en el cerebro, lo va estructurando y reestructurando mediante su codificación en la corteza; así se organiza la actividad consciente del niño, se puede decir que el cerebro se socializa y se dirige a ser el aparato psíquico de la personalidad. A partir del reflejo de este tipo de información se forma la actividad psíquica consciente, lo que no quiere decir, que la actividad psíquica inconsciente traída por el niño deje de existir, lo que ocurrirá es que será "subsumida" y sometida al nivel consciente, el que lo reestructurará. Dejamos constancia que no es lo mismo la actividad inconsciente planteada por la teoría psicobiológica dialéctica y la propuesta por Freud, aquella procesa información psíquica en el último nivel de la determinación epigenética, en la gestación, y en los primeros años de vida; se caracteriza porque su actividad busca la satisfacción de las necesidades llamadas primarias y por ello 
"tiene su base de desarrollo en los aspectos superficiales del medio ambiente inmediato que el niño encuentra y registra al momento de nacer" (Ortiz P. "Psicobiología humana IV"), en cambio el psicoanálisis presenta una propuesta centrada en una topografía del psiquismo predeterminada y donde hay una supremacía de lo inconsciente sobre lo consciente durante toda la existencia del individuo.

La información, en la determinación epigenética, se encuentra al interior del ser vivo (proceso de gestación), en sus cuatro niveles, en cambio en la determinación sociocinética la información social está fuera del individuo y éste la incorpora para llegar a ser persona. Se entiende que la información social es creada por la sociedad y es el niño, que para llegar ser persona, debe incorporarla; es decir en su devenir sociocinético el hombre, en la práctica, construye al hombre.

En la sociocinesis el sujeto codifica una cantidad y cualidad de información social que le determinará una cantidad y cualidad de información psíquica consciente, reestructurando permanentemente su neocórtex, reflejando de esta manera el momento histórico que le ha tocado vivir y donde formará su personalidad. Para Lucien Séve se constituye la formación social de la individualidad histórica social.

Cómo se definiría la conciencia de acuerdo a la teoría psicobiológica dialéctica: "Es la información social codificada en el neocórtex y transformada en información psíquica consciente". Esta concepción de la conciencia requiere una explicación. En primer lugar y partiendo de la historia de la psicología, sabemos que en la psicología soviética al cerebro se le consideró "la base material del psiquismo", o como soporte de la actividad psíquica; en la propuesta de Ortiz el cerebro mismo es la conciencia, específicamente el neocortex, cuya actividad más compleja y "altamente organizada" es la actividad psíquica consciente; la conciencia es vista como un sistema donde el neocortex es la estructura y su actividad es la información psíquica consciente; esta separación de estructura y actividad es con fines únicamente explicativos, no se les pueden separar se les tiene que comprender como una estructura activada o como una actividad estructurada, lo que nos obliga a pensar que la actividad consciente no es algo inmaterial sino todo lo contrario. Con este planteamiento se elimina toda posibilidad de dualismo. Además, desde esta óptica, sí podemos decir que la conciencia es "el movimiento de la materia más altamente organizada" y que se concretará en la actividad estructurada del neocortex por el reflejo de la información social.

No se piense que la corteza recibirá la información de manera pasiva, que actúa como un papel en blanco y que la información social se escribirá en ella, no es así. El recién nacido trae una corteza no estructurada para vivir en sociedad, construida durante la determinación epigenética, es consecuencia del actuar de los niveles genético, tisular, orgánico e inconsciente; lo que indica que al reflejar la información social, la corteza cerebral actúa con toda la información epigenética que posee, reestructurándose a partir de aquella; esta interactuación de lo epigenético con lo sociocinético, a nuestro modo de ver, determina la singularidad de la persona. La conciencia concebida dentro de la teoría de Ortiz tiene tres componentes: Afectivo-Emocional, ubicado en la corteza paralímbica; CognitivoProductivo que se encuentra en la corteza parietotemporooccipital y el Conativo-Volitivo en la corteza del lóbulo frontal, en el área dorsolateral. Estos componentes, de manera paulatina, durante la sociocinesis, en la juventud, van a constituir las partes integrantes de la personalidad: el temperamento, el intelecto y el carácter respectivamente. 


\section{CONCIENCIA BÁSICA}

Mediante el proceso de sociocinesis se determina la conciencia con la codificación de la información social, ésta produce modificaciones en el neocortex, haciéndolo cambiar o evolucionar en un proceso constante de estructuración y reestructuración que le permite al individuo vivir dentro de la sociedad; pero no solo el cerebro cambia sino toda la persona de forma integral, desde el nivel consciente hasta el genético, esto se demuestra durante la filogenia de la especie Homo. Esta evolución o cambio en el neocórtex determinan también cambios a nivel consciente en el niño que pueden ser vistos como desarrollo o no desarrollo de la persona, pero de todas maneras se produce una evolución o cambios con la determinación sociocinética, en la conciencia.

Es decir, una conciencia evoluciona desarrollando o simplemente cambiando, este hecho se debe al tipo de información social que procesa el sujeto, la que le permite, reitero, el desarrollo o solamente el cambio o evolución. Es correcto, entonces, llamar PSICOLOGÍA EVOLUTIVA y no PSICOLOGÍA DEL DESARROLLO, porque no todo cambio implica desarrollo; es decir, toda la información que refleja la persona no le va a implicar un desarrollo personal, le hará ver el mundo de diferente manera, le modificará su actuación personal, pero no quiere decir que el individuo está en camino a ser persona.

El desarrollo personal o el camino a ser persona tienen su eje principal en el tipo de información que el individuo refleje, ésta debe estructurarlo y organizarlo hacia características personales de dignidad, autonomía e integridad, de tal forma que la información social afectiva, cognitiva y conativa debe encaminar al individuo a dichas características.

Después de la determinación epigenética, el recién nacido tiene una corteza cerebral que le permite reflejar la información social y convertirla en psíquica consciente, es decir nace como humano en camino de ser persona; paulatinamente y conforme aumenta en cantidad y calidad la información psíquica, se constituye la conciencia. Retomando la idea de los cambios que se producen con la sociocinesis, desde la niñez se va conformando la conciencia (en sus tres componentes: afectivo-emotivo, cognitivo-productivo, conativo-volitivo) que va estructurándose y reestructurándose hasta conformar los tres componentes; éstos no tienen un proceso de formación igual, pero debe llegar el momento en que el individuo los tiene relativamente constituidos y se dice que es una personalidad con su respectivo aparato psíquico, la conciencia; relativa es porque ésta siempre se modificará por el permanente reflejo de la información que le determina su rumbo y evolución. El proceso de construcción y organización de la conciencia se deben a dos tipos de posibilidades, que se le presentan a la persona.

\section{Posibilidad de la persona para acceder a la información social en cantidad y cualidad; la información social que la sociedad le posibilita a acceder a la persona}

En un caso son las posibilidades que tiene la persona para captar información social en cantidad y cualidad de la sociedad en que vive; la segunda posibilidad está definida por la sociedad al presentarle una cantidad y cualidad de información social al individuo para la estructuración y actividad de la conciencia, en ambos casos estas posibilidades tienen como soporte las condiciones económico-sociales propias e individuales del sujeto, ambas 
posibilidades no se dan por separado, pues están dentro de un mismo contexto social, la relación que presentan ambas tiene carácter recíproco. De esta manera entendemos, la determinación social de la conciencia y, por ende, de la personalidad.

La cantidad y cualidad, que son elementos generales de la información social, juegan rol importante en la configuración de la conciencia, ellas están absolutamente ligadas a las condiciones sociales en que vive la persona y la oportunidad que dicha condición les brinde para la captación de la información social. Podemos afirmar que la clase social donde pertenece el individuo está estructurada por una información social que la determina y la organiza, a su vez se constituye en la base de desarrollo para la estructuración de la conciencia de los individuos que forman dicha clase.

Hemos dicho que la evolución de la conciencia está ligada a dos aspectos generales: la cualidad y la cantidad de información; por cualidad de la información social se entiende, de manera específica, aquella que al reflejarse en el sujeto le es favorable o desfavorable para su desarrollo conciencial y personal, en el primer caso, cuando es favorable y que lo lleva a las características de dignidad, autonomía e integridad, le llamaremos información social de desarrollo (ISD); en el segundo, información social de evolución (ISE). Reiteramos que entendemos por desarrollo personal, al proceso por el cual el individuo se hace digno, autónomo e íntegro, que va modificando sus componentes concienciales y llegará a una conatividad cuya formación tiene como fondo la moral donde los valores ligados a la integridad, honestidad, solidaridad, etc., son los organizadores de la conciencia y por lo tanto de la persona.

El componente conativo filo y ontogenéticamente se constituye en último momento y es el que, verdaderamente, nos separa del reino animal, por lo que su estructuración concretiza el desarrollo personal. Además hay que considerar que el componente conativo es consecuencia de la integración, en el lóbulo frontal, de parte de los componentes afectivo y cognitivo y su estructuración se produce a finales de la adolescencia e inicios de la juventud, momento en que termina de constituirse la corteza del lóbulo frontal. Por todas estas ideas es que el desarrollo lo orientamos hacia el plano conativo-volitivo; dicho de otra forma, la 1SD organiza y orienta a la conciencia, conjugando sus tres componentes, hacia valores propios de la persona; la afectividad, que tiene su base de desarrollo en la información social tradicional, se estructurará en base al afecto proporcionado en la niñez, conformando sentimientos alturados; en cuanto al componente cognitivo, la 1SD permitirá el desarrollo del pensamiento crítico, la reflexión analítica, pasar de una forma fenoménica de reflejar la realidad a otra sustancial o esencial, así mismo permitirá una visión holística de la realidad, dará la mayor variedad de conocimientos de lo natural como de lo social, buscando la esencia de las cosas y la verdad. Se entenderá que los componentes de la conciencia no están separados, al contrario tiene una absoluta interacción dialéctica, aquí los separamos por razones explicativas y se puede decir, por ejemplo, que se piensa sintiendo en la motivación, o, la motivación es pensada y sentida, o, siento pensando porque tengo una motivación. La información social de evolución, ISE, es aquella que estructura la conciencia sin determinar un desarrollo personal, se caracteriza el componente cognitivo por ser fenoménica no puede llegar a ver las causas de las cosas, es limitada en conocimientos y la cognitividad se sustenta en el sentido común, percibe la realidad de manera parcelada; en el campo afectivo los sentimientos están distorsionados, se desconoce el amor y la 
ternura, en cambio se dirige la afectividad a lo inesencial a lo vacío, los sentimientos están dirigidos al individualismo al egoísmo, hay predominio hacia la mentira; donde más se nota el poco desarrollo de la conciencia es en el componente conativo-volitivo, la moral no es un valor, hay predominio de lo inmoral y hasta se llega a lo antimoral.

Aquí debemos detenernos y retomar lo que dijimos sobre el desarrollo personal, el componente conativo de la conciencia es propio de la personalidad, ningún ser ni el más superior del reino animal lo posee y es el que diferencia a la persona de todos los demás seres; se puede decir que los animales aprenden o que tienen sentimientos (apego) pero nunca motivaciones, ideales o moral: esta es la razón por la que es ideológico y no científico proponer que el psiquismo del animal puede ser comparable con la actividad consciente de la persona, la misma que ha tomado una inmensa distancia con respecto a los animales y su epigénesis está totalmente sometida a la sociocinesis. Entonces, el desarrollo personal está dirigido hacia la construcción del componente conativo, con las cualidades o características que se han mencionado arriba.

Cuando la conciencia solo evoluciona, el individuo presenta un mínimo de información social para vivir dentro de la sociedad, la mayor cantidad de ella no le es válida para desarrollarse como persona. Aquí veremos el otro aspecto general de la conciencia, la cantidad de la información social; se entenderá que no toda información social, a pesar que la ha creado el hombre, permite el desarrollo personal; sin embargo la persona puede poseer un inmenso bagaje de información social pero ella no la deja desarrollarse e ir superándose y llegar a ser persona. Un individuo con este grado de desarrollo de conciencia se desenvuelve dentro de la sociedad de forma limitada. Pongamos un ejemplo: un individuo tiene una cultura futbolística amplia, su permanente motivación está en ese deporte, su Tendencia Orientadora de la Personalidad, TOP, (Gonzáles Rey Fernando, Psicología Principios y Categorías) está dirigida únicamente hacia el fútbol, pero él se desenvuelve dentro de la sociedad con un mínimo de información social que le permite sobrevivir, con ella trabaja, se relaciona, hace vida familiar, etc.; es la información social de ese deporte la que estructurará y organizará la conciencia. En otro caso no es obligatorio que se posea una TOP, puede ocurrir que solo tenga información social sustentada en tradiciones, costumbres, o lo que la vida cotidiana de manera empírica enseña, en ese caso, el individuo tiene como base de desarrollo aquellas informaciones porque son las que van a organizar su conciencia para sobrevivir a diario. La cantidad de información social está relacionada con su calidad, la combinación de ambas se puede deducir el grado de desarrollo de la conciencia.

En cuanto a la información social que determina sólo una evolución (ISE), por deducción lógica tendrá las características contrarias a la ISD, aquella impide el desarrollo personal ésta lo determina. La información social que no permite el desarrollo personal la encontramos diario y en inmensas cantidades y cualidades, solo basta encender la TV y observar sus programas, por ejemplo los que trasmiten las estaciones locales los días sábados en la noche. En los medios de divulgación, en general, la información social es ajena a una ISD, por el contrario lleva al ciudadano por mundos completamente impropios, fuera de todo contexto, imposibilitándolo de pasar de un reflejo de la realidad mínimo a otro superior. Tanto en la niñez como en la adolescencia, los medios de divulgación, específicamente la televisión, forman parte de la llamada educación informal que durante el día está más tiempo con ellos 
que la propia escuela. En esencia la educación informal no desarrolla la personalidad, desvía su posibilidad de mejorar. La misma escuela, si mantiene las características memorísticas, superfluas, autoritarias, dogmáticas, no cumple con el desarrollo personal, inclusive las condiciones precarias en que se realiza la educación formal no contribuye a que el niño o adolescente logre cumplir con los objetivos educativos a los que su edad le corresponde.

El aspecto de la información, cantidad, no puede ser estudiada solo, está íntimamente ligada a la cualidad. Lo ideal sería que en una sociedad abunde la ISD pues llevaría inequívocamente a un desarrollo personal y por supuesto a una conciencia individual también desarrollada; pero lo que ocurre es lo contrario; la sociedad presenta una información social negativa abundante como la que existe en nuestra realidad, entonces podemos inferir que la conciencia, en los peruanos mayoritariamente, será no desarrollada.

Aparentemente este planteamiento sobre la relación entre la información social y la sociedad parece mecanicista, pero desde el momento en que la propia sociedad es la que produce la información y ella misma la capta deja toda posibilidad de ser mecánica dicha relación; además hay que recordar que no todo individuo interioriza la IS de la misma forma, esto ya lo hemos explicado anteriormente, la interactuación de la epigénesis con la sociocinesis determina la individualidad y singularidad personal, por ejemplo el caso excepcional de la resiliencia. Luego las condiciones materiales de existencia son esenciales para alcanzar un grado de desarrollo de la conciencia, es decir que una sociedad donde las clases se organizan con un tipo de información social a partir de sus condiciones materiales se distinguen, por ejemplo, por el acceso a la información social cultural que cuando que se codifica en el neocortex se traduce como información psíquica cognitiva. También de la relación de lo individual a lo social y de lo social a lo individual al participar ambas partes de una manera refleja, se elimina el posible mecanicismo, fundamentalmente al entender la contradicción de la epigénesis con la sociocinesis. En fin son múltiples las razones para negar el mecanicismo que aparentemente se presenta, y más bien sí encontramos una dialéctica claramente expresada en este proceso de personalización

De acuerdo con todo lo dicho, podemos decir que la conciencia en su devenir histórico personal pasa por un permanente cambio, lo que nos lleva a pensar que presenta grados en su desarrollo. El primer grado estaría dado por aquellas personas que reproducen el sistema de forma fenoménica, unilateral, sesgadamente, etc., por poseer una información social mínima cualitativa y cuantitativamente, con lo cual pueden subsistir; a este grado lo denominamos "conciencia básica"; en ella puede haber gran cantidad de información pero la calidad y también la cantidad puede ser mínima abundando la ISE, estos criterios serían los que caracterizaran a este grado de desarrollo de la conciencia. Cuando la persona ha reflejado predominantemente ISD y en la práctica demuestra que ha hecho suyo este reflejo o ese tipo de información, decimos que presenta un grado de desarrollo superior denominado "autoconciencia"; ésta se caracteriza por tener elementos contrarios a los de la conciencia básica: ve más allá del fenómeno, es holística, la persona se ubica dentro del proceso productivo, no es conservadora busca el cambio social, analiza y critica sus condiciones materiales de existencia. En síntesis, podemos decir que conciencia básica es un grado de desarrollo de la conciencia, cuya naturaleza es el mínimo cuanti y cualitativamente de ISD con la que puede vivir una persona. 
Como lo hemos sugerido antes, en el Perú existe un elevado porcentaje de población con conciencia básica y ello sería la explicación psicológica de por qué estamos en las condiciones de crisis permanente, con gobiernos que engañan constantemente al pueblo, de tener la profunda miseria en las mayorías de peruanos. Pues con la información social se manipula la conciencia y se mantiene a todo un pueblo como si estuviera dentro de una burbuja sin poder salir de ella.

\section{REFERENCIAS BIBLIOGRÁFICAS}

1. Gonzáles Rey Fernando (1989). Psicología principios y categorías. Ciencias Sociales, La Habana.

2. Ortiz Cabanillas Pedro (2004). Introducción a una psicobiologia del hombre. Lima: UNMSM, Facultad de Medicina.

3. (2004). El nivel consciente de la actividad personal. Lima: UNMSM, Facultad de Medicina.

4. Seve Lucien (1975). Marxismo y teoría de la personalidad. Buenos Aires: Amorrortu editores. 